\title{
Study on the Effect of Temperature on the Equipment Delay of Two-way Satellite Time and Frequency Transfer
}

\author{
Guoyong Wang1, a, Yansong Meng ${ }^{1}$, Lang Bian¹ and Ya Liu² \\ ${ }^{1}$ China Academy of Space Technology (Xi'an), Xi'an 710000, China; \\ ${ }^{2}$ National Time Service Center, Chinese Academy of Sciences, Xi'an 710600, China. \\ awangguoyong321@163.com
}

\begin{abstract}
Keywords: Two-way Satellite Time and Frequency Transfer; Equipment Delay, Negative Temperature Coefficient.
\end{abstract}

\begin{abstract}
Two-way Satellite Time and Frequency Transfer (TWSTFT) is one of the most important ways to achieve high-precision synchronization between stations. In the TWSTFT, the equipment delay changes with temperature. So the equipment delay caused by TWSTFT transmission and reception equipment is not easy to calibrate, which is the most important factor affecting the accuracy of time synchronization between stations. In this paper, the influence of temperature on the delay of the modem and whole device of TWSTFT were studied. The experimental results show that the indoor modem is not sensitive to temperature, the negative temperature coefficient is about $6 \mathrm{ps} /{ }^{\circ} \mathrm{C}$; the negative temperature coefficient of whole TWSFTF equipment delay difference is about $18 \mathrm{ps} /{ }^{\circ} \mathrm{C}$.
\end{abstract}

\section{Introduction}

TWSTFT is one of the most important techniques for comparing time scales and atomic frequency standards with an uncertainty in time of less than $1 \mathrm{~ns}$ and relative uncertainty for frequency of about $10^{-15} /$ day ${ }^{[1]}$. However, the different signal delays were involved in TWSTFT system. So it is necessary to calibrate those delays ${ }^{[2-5]}$.

In order to calibrate systematic difference any TWSTFT links, we developed two same mobile stations. Every mobile station is mainly consist of a modem and a very small aperture terminal (VSAT). Where, Satellite Time and Ranging Equipment (SATRE) was used as a modem that made in Germany Teletech Company. In this paper, we study on the effect of temperature on the equipment delay of TWSTFT ${ }^{[6-7]}$.

\section{The Principle of TWSTFT}

According to basic principles of TWSTFT, the timescale difference TS(1)-TS(2) between 1 and 2 sites is presented as follows ${ }^{[8]}$ :

$\operatorname{TS}(1)-\operatorname{TS}(2)=0.5\{[\operatorname{TIC}(1)-\operatorname{TIC}(2)]+\Delta \tau\}$

$\Delta \tau=\Delta \mathrm{TR}+\Delta \mathrm{TL}+\Delta \mathrm{SP}+\Delta \mathrm{SC}$

Where, TIC(i) represents reading of counter at i station, the $\Delta \tau$ stands for nonreciprocal residual effects. It includes the delay differences due to: 1) inside each earth station including transmission and reception paths( $\Delta \mathrm{TR}) ; 2$ ) the propagation in the atmosphere for up and down links( $\Delta \mathrm{TL}) ; 3)$ the transponder path in the geostationary satellite( $\Delta \mathrm{SP})$; and 4) the earth rotation (Sagnac effect: $\triangle \mathrm{SC}$ parameter); The first parameter seems to be the most important source of error which is developed in (3)

$\Delta \mathrm{TR}=[\mathrm{TX}(1)+\mathrm{RX}(2)]-[\mathrm{TX}(2)+\mathrm{RX}(1)]$

Where TX (i) and RX (i) respectively represent the delay of transmission and reception paths at $\mathrm{i}$ station. 


\section{The Effect of Temperature on the Equipment Delay}

\subsection{The Effect of Temperature on the Equipment Delay of modem}

Experimental principle was shown in Figure 1. The atomic clock generates $1 \mathrm{pps}$ and $10 \mathrm{MHz}$ signals. Through the distribution amplifier, those signals were sent to the two modems as reference signals. In this experiment, two modems from Timetech was used, named respectively SATRE 1 and SATRE 2. The transmission channel RFOUT of SATRE 1 and the reception channel RFIN of SATER 2 were linked by two cables and an attenuator. Also, the transmission channel RFOUT of SATRE 2 and the reception channel RFIN of SATER 1 are also linked by two cables and an attenuator. Four cables of connecting the RF input and output ports of SATRE 1 and 2 are same long. SATRE 1 and SATRE 2, respectively obtain measurements TIC1 and TIC2. Where TIC1 and TIC2 represent SATRE1 and 2 reception channel measurement value, which includes transmit delay, receive delay, cable delay, attenuator delay. As shown in equation (3), we are not concerned with the transition delay or reception delay of a single ground station in TWSTFT, but the equipment delay difference between the two ground stations. This experiment uses the equipment delay difference between SATRE 1 and 2 to study on the delay stability of TWSTFT based on temperature.

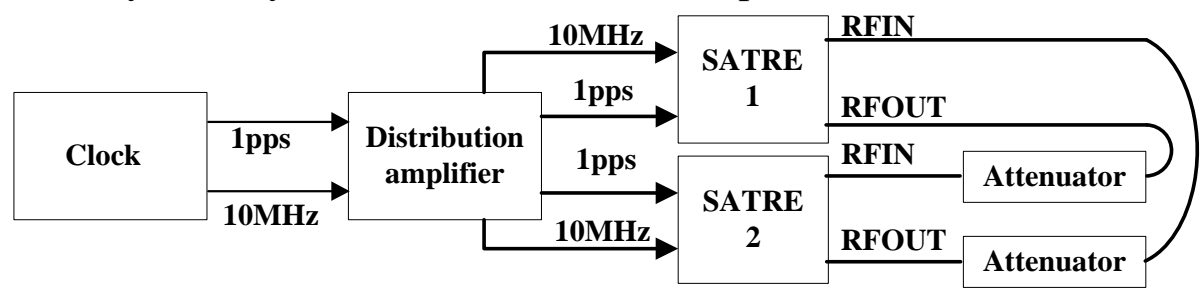

Fig. 1 The structure of measurement system on modem equipment delay difference

Figure 2 is equipment delay difference of modem. The blue lines are the raw data, and the red lines are the result after the least squares fitting. The mean of equipment delay difference is $1.1048 \mathrm{~ns}$ and the standard is $0.0111 \mathrm{~ns}$. The duration of the experiment is 1 hour, the temperature drops by $3.5^{\circ} \mathrm{C}$. The variation of the red line is about $0.02 \mathrm{~ns}$, and the negative temperature coefficient of equipment delay difference is about $6 \mathrm{ps} /{ }^{\circ} \mathrm{C}$. The experimental results show that equipment delay difference of the modem is relatively stable.

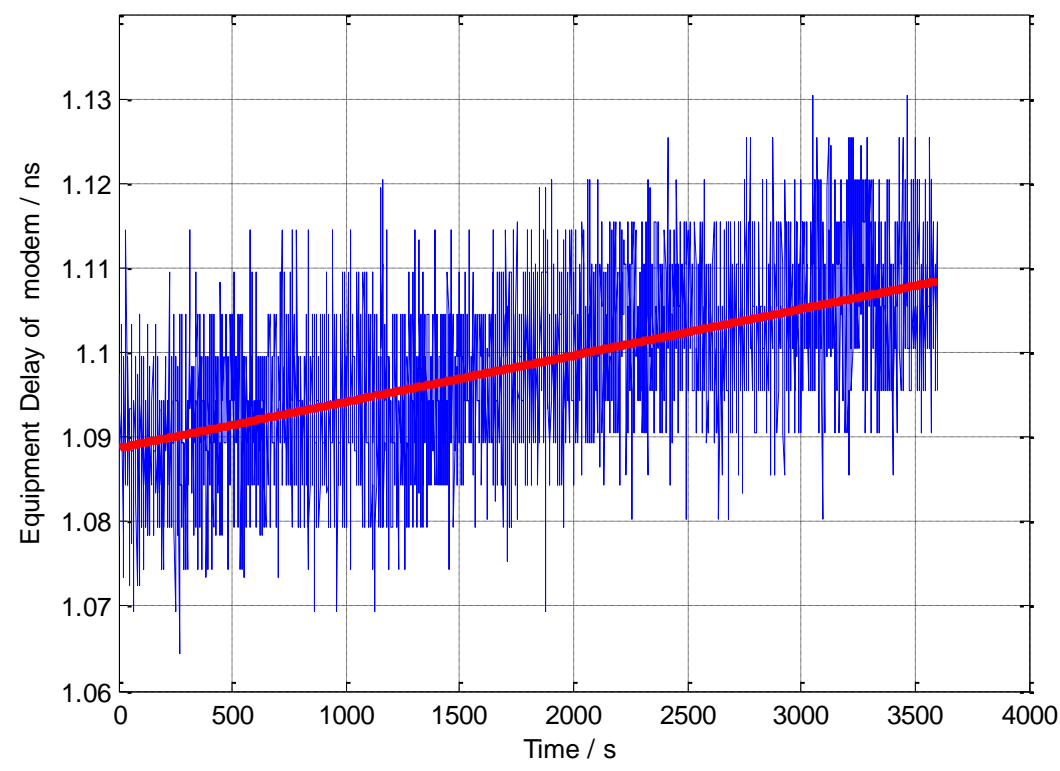

Fig. 2 The equipment delay difference of modem

\subsection{The Effect of Temperature on the Equipment Delay of TWSTFT}

The structure of zero-baseline time delay measurement system illustrated in Fig. 3 is used to measure the equipment delay difference $\triangle$ TR between two mobile stations. The method is that two 
mobile stations are both placed in the same site and the same clock was used. So we can assume TS(1) $-\mathrm{TS}(2)=0, \triangle \mathrm{TL}=0, \triangle \mathrm{SP}=0, \triangle \mathrm{SC}=0$. So $\triangle \mathrm{TR}=0.5[\mathrm{TIC}(2)-\mathrm{TIC}(1)]$

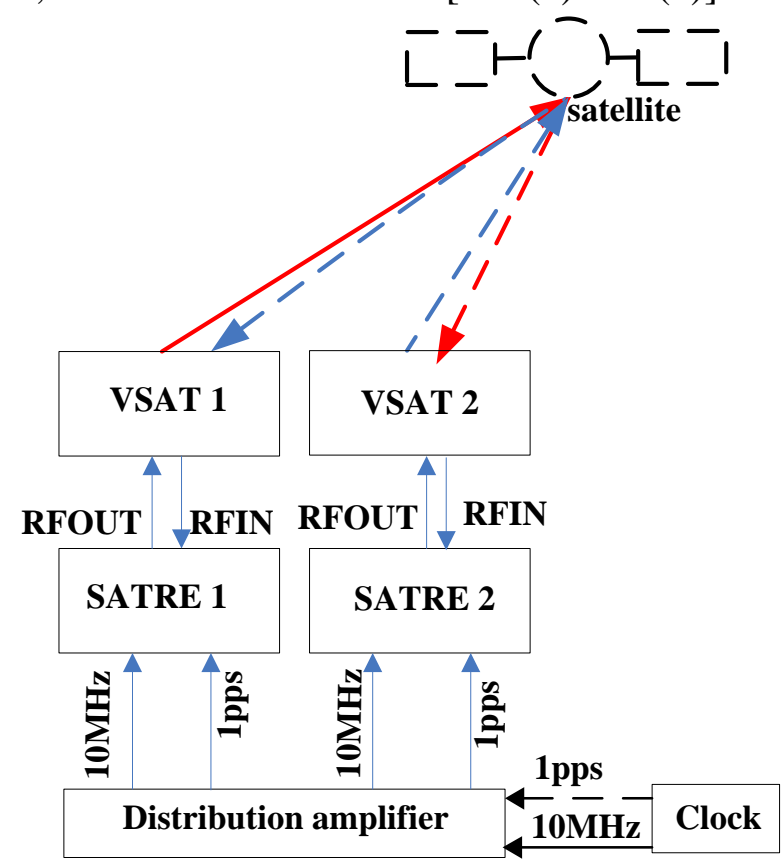

Fig. 3 The structure of zero-baseline time delay measurement system

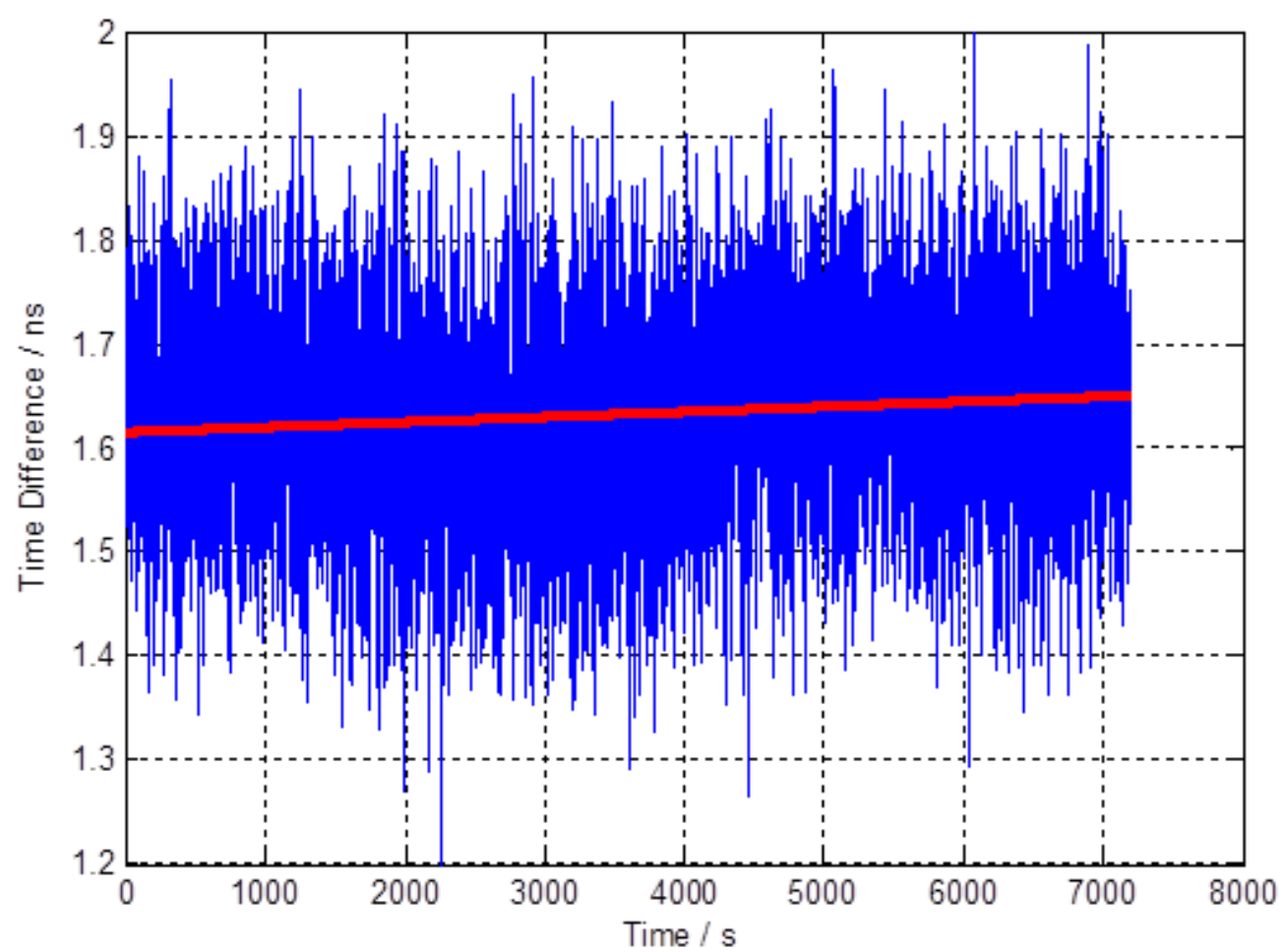

Fig. 4 The equipment delay difference of TWSTFT between two mobile stations

Figure 4 shows the equipment delay difference of TWSTFT between two mobile stations about two hours. It reflects the relationship between temperature and equipment delay difference. The blue line is the original data, the red line is the result after the least squares fitting. The mean of equipment delay difference is $1.6823 \mathrm{~ns}$ and the standard is $0.1407 \mathrm{~ns}$. When the temperature of the outdoor device is reduced by $2{ }^{\circ} \mathrm{C}$, the change of the red line is about $0.035 \mathrm{~ns}$, and the negative temperature coefficient of whole TWSFTF equipment delay difference is about $18 \mathrm{ps} /{ }^{\circ} \mathrm{C}$. And the negative temperature coefficient of the modem equipment delay difference is about $6 \mathrm{ps} /{ }^{\circ} \mathrm{C}$. So the negative temperature 
coefficient of equipment delay difference of outdoor equipment is about $12 \mathrm{ps} /{ }^{\circ} \mathrm{C}$, which is the main factor of equipment delay variation.

\section{Conclusion}

In this paper, based on two mobile stations, the effect of temperature on the equipment delay of TWSTFT was studied. The influence of temperature on the equipment delay difference of the modem and whole device of TWSTFT were studied. The experimental results show that the indoor modem is not sensitive to temperature, the negative temperature coefficient is about $6 \mathrm{ps} /{ }^{\circ} \mathrm{C}$; outdoor equipment is sensitive to temperature, the negative temperature coefficient is about $12 \mathrm{ps} /{ }^{\circ} \mathrm{C}$. The negative temperature coefficient of whole TWSFTF equipment delay difference is about $18 \mathrm{ps} /{ }^{\circ} \mathrm{C}$.

\section{Acknowledgments}

This work was supported by the National Natural Science Foundation of China (Nos. 61627817).

\section{References}

[1]. A Bauch, J Achkar, S Bize, etc. Comparison between frequency standards in Europe and the USA at the 10-15 uncertainty level. Metrologia 43 (2006), 109 -120.

[2]. D. Piester, A. Bauch, J. Becker, et al. Time Transfer between USNO and PTB: Operation and Calibration Results[C]. 35th Annual Precise Time and Time Interval Meeting, 2003, 93-102.

[3]. D. Piester, J. Achkar, J. Becker etc. Calibration of Six European TWSTFT Earth Stations Using a Portable Station [J], Proceedings of the 20th European Frequency and Time Forum, Braunschweig, Germany (2006), 2006,460-467.

[4]. D. Piester, A. Bauch, L. Breakiron etc. Time transfer with nanosecond accuracy for the realization of International Atomic Time [J]. Metrologia, 2008, 45 (2), 185-198.

[5]. D. Piester, R. Hlavac', J. Achkar etc. Calibration of Four European TWSTFT Earth Stations with a Portable Station through INTELSAT 903 [J], Proceedings of the 19th European Frequency and Time Forum, Besancon, France, 2005, 354-359.

[6]. Guoyong Wang, "Research on Systematic Errors Calibration Method Based on Double Mobile Stations in Two-way Satellite Time Transfer" [D], The University of Chinese Academy of Sciences, 2015.

[7]. Guoyong Wang, Ya Liu, Xiaohui Li, Chen Shi, "Research Progress on TWSTFT Mobile Calibration Station". Lecture Notes in Electrical Engineering, Vols.340, 2015, pp415-424.

[8]. Guoyong Wang, Ya Liu, Xiaohui Li, Ruifang Dong, “A new method of time difference calibration of TWSTFT earth station based on two portable stations". 2014 IEEE International Frequency Control Symposium (IFCS), 2014, pp1-5. 